\title{
Combined optical coherence tomography and hyperspectral imaging using a double-clad fiber coupler
}

Robin Guay-Lord

Xavier Attendu

Kristen L. Lurie

Lucas Majeau

Nicolas Godbout

Audrey K. Ellerbee Bowden

Mathias Strupler

Caroline Boudoux 


\title{
Combined optical coherence tomography and hyperspectral imaging using a double-clad fiber coupler
}

\author{
Robin Guay-Lord, ${ }^{\mathrm{a}}$ Xavier Attendu, ${ }^{\mathrm{a}}$ Kristen L. Lurie, ${ }^{\mathrm{b}}$ Lucas Majeau, ${ }^{\mathrm{a}}$ Nicolas Godbout, ${ }^{\mathrm{a}, \mathrm{c}}$ \\ Audrey K. Ellerbee Bowden, ${ }^{\mathrm{b}}$ Mathias Strupler, ${ }^{\mathrm{a}}$ and Caroline Boudoux ${ }^{\mathrm{a}, \mathrm{c}, *}$ \\ á́́cole Polytechnique Montreal, Department of Engineering Physics, C.P. 6079 Succ. Centre-ville, Montréal, Canada \\ ' Stanford University, E.L. Ginzton Laboratory, 350 Serra Mall, Packa Road, Room 361, Stanford, California 94305, United States \\ 'Castor Optics, 5155 Avenue Decelles 1251, Pavillon J-Armand Bombardier, Montréal, Québec H3T 2B1, Canada
}

\begin{abstract}
This work demonstrates the combination of optical coherence tomography (OCT) and hyperspectral imaging (HSI) using a double-clad optical fiber coupler. The single-mode core of the fiber is used for OCT imaging, while the inner cladding of the double-clad fiber provides an efficient way to capture the reflectance spectrum of the sample. The combination of both methods enables three-dimensional acquisition of the sample morphology with OCT, enhanced with complementary molecular information contained in the hyperspectral image. The HSI data can be used to highlight the presence of specific molecules with characteristic absorption peaks or to produce true color images overlaid on the OCT volume for improved tissue identification by the clinician. Such a system could be implemented in a number of clinical endoscopic applications and could improve the current practice in tissue characterization, diagnosis, and surgical guidance. (๑) The Authors. Published by SPIE under a Creative Commons Attribution 3.0 Unported License. Distribution or reproduction of this work in whole or in part requires full attribution of the original publication, including its DOI. [DOI: 10.1117/1.JBO.21.11.116008]
\end{abstract}

Keywords: optical coherence tomography; hyperspectral imaging; dual-modality; double-clad fiber; double-clad fiber coupler. Paper 160454LPR received Jul. 29, 2016; accepted for publication Oct. 7, 2016; published online Nov. 9, 2016.

\section{Introduction}

Over the last decade, optical coherence tomography (OCT) has developed into a significant clinical imaging modality. For example, it has become a primary technique to investigate the eye $^{1}$ and blood vessels, ${ }^{2}$ and many applications are emerging in laryngology, ${ }^{3}$ gastroenterology, ${ }^{4,5}$ dentistry, ${ }^{6}$ and many other fields of medicine. OCT has the ability to image organs in threedimensions (3-D), with a resolution of a few micrometers, and a penetration depth of several millimeters at video rate acquisition speeds. ${ }^{7}$ However, while OCT is a very powerful technique to assess sample morphology, it gives access to little molecular information.

In contrast, hyperspectral imaging (HSI) is an imaging method that provides two-dimensional images of a sample in multiple wavelength bands. ${ }^{8}$ A reflectance (or fluorescence) spectrum is associated with each pixel and conveys information on molecules present locally, but without depth discrimination. The technique has been successfully applied in cancer detection, ${ }^{9-11}$ diagnosis of circulatory and ${ }^{12-14}$ retinal $^{15-17}$ diseases, and surgical guidance. ${ }^{18-20}$

As OCT and HSI give access to complementary information, their combination could improve their prowess in a clinical context. Spectroscopic OCT is one way to combine both techniques and consists of using the same laser and detectors for OCT and spectroscopy. ${ }^{21-24}$ Such implementation requires different signal processing of the same original data to obtain depth-resolved hyperspectral images and OCT images. ${ }^{25}$

*Address all correspondence to: Caroline Boudoux, E-mail: caroline.boudoux@ polymtl.ca
However, few sources suitable for OCT have the large bandwidth required for meaningful spectral analysis. The use of such sources is also challenging due to the dispersion management required in OCT. Furthermore, to allow millimeter-long penetration depth, the central wavelength of the OCT source must be in the near-infrared region (NIR). However, many biological absorbers show fewer spectral variations in the NIR than in the visible range, and would therefore not be identifiable using this spectral band.

In this paper, we describe an alternative strategy to implement HSI and OCT using two different sets of light sources and detectors with both channels combined into a single imaging probe. This allows choosing the spectral bands for OCT and HSI independently. While other groups have reported combining OCT and HSI in free-space systems, ${ }^{26,27}$ we present an allfiber implementation based on a double-clad fiber (DCF). DCFs support two communication channels: a single-mode channel in the core and a multimode channel in the inner-cladding. Using a double-clad fiber coupler (DCFC), it is possible to address both channels efficiently. ${ }^{28-30}$ The viability of dual-modality systems based on DCFCs was already demonstrated in systems coupling OCT and fluorescence, ${ }^{31,32}$ or OCT and laser marking. ${ }^{33}$ The robustness and compactness of these all-fiber systems facilitate their integration into standard clinical instruments. ${ }^{28}$

We herein present the first combination of HSI and OCT in a single-optical fiber component using a DCFC. OCT acquisition is realized with a swept-source system and uses the DCF core for illumination and collection of the backscattered photons. For HSI acquisition, an external white light source is used to illuminate the sample. The reflected light is collected by the DCF inner-cladding, extracted by the DCFC into a multimode (MM) fiber and sent to a spectrometer for detection. The performance 
of the system is demonstrated by imaging healing epithelial tissue on the hand of a volunteer.

\section{Methods}

\subsection{Imaging Setup}

The imaging setup is shown in Fig. 1. It is based on a commercial OCT system (OCM1300SS, Thorlabs, New Jersey) and uses a wavelength-swept laser centered at $1325 \mathrm{~nm}$ with a bandwidth of $100 \mathrm{~nm}$, a coherence length of $6 \mathrm{~mm}$, and an axial scanning (A-line) rate of $16 \mathrm{kHz}$. This system was modified to enable HSI by inserting a DCFC in the sample arm. The DCFC (DC1300LEB, Thorlabs, New Jersey) is made by fusing and tapering a DCF and an MM fiber. ${ }^{29}$ The diameters of the DCF core, inner-cladding, and outer-cladding are 9, 105, and $125 \mu \mathrm{m}$, respectively. The MM fiber has a core diameter of $200 \mu \mathrm{m}$. The cladding mode extraction efficiency of the DCFC (cladding mode transmission from port $\mathrm{S}$ to port $\mathrm{B}$ ) is higher than $60 \%$ while the injection loss of the core signal (core mode losses from port A to port $\mathrm{S}$ ) is lower than $0.5 \mathrm{~dB}$.

The output of the wavelength-swept laser is sent to an external Michelson type interferometer module (INT-MSI-1300, Thorlabs, New Jersey) that splits the light into the sample and reference arms and also includes a $15-\mathrm{MHz}$ balanced detector. An SMF-28 fiber is connected to the sample arm output of the interferometer module using an FC-APC connector and fusionspliced at the other end to the DCF input of the DCFC (port A). The DCF output (port $S$ ) of the DCFC is then collimated with a 16-mm focal length fiber collimation lens (C2: FC260APC-C, Thorlabs, New Jersey). A dual-axis galvanometer is used to steer the beam (G: GVS002, Thorlabs, New Jersey). An uncoated, 50$\mathrm{mm}$ focal length planoconvex lens (L: LA1131, Thorlabs, New Jersey), placed at one focal length of the galvanometer, is used to focus the beam on the sample for a telecentric scan. The light backscattered by the sample is collected by the fiber core and cladding, but only the signal collected in the core can travel back to the OCT interferometer. Light in the cladding is either transferred to the MM branch of the DCFC or lost at the splice between the DCF and the SMF-28 fiber. The reference arm is composed of an SMF-28 fiber that matches the sample arm dispersion. Since the double-clad and SMF-28 fibers have the same dispersion characteristics, the length of the SMF-28 fiber in the reference arm is simply the sum of all SMF-28 and DCF segments in the sample arm. No dispersion compensation method

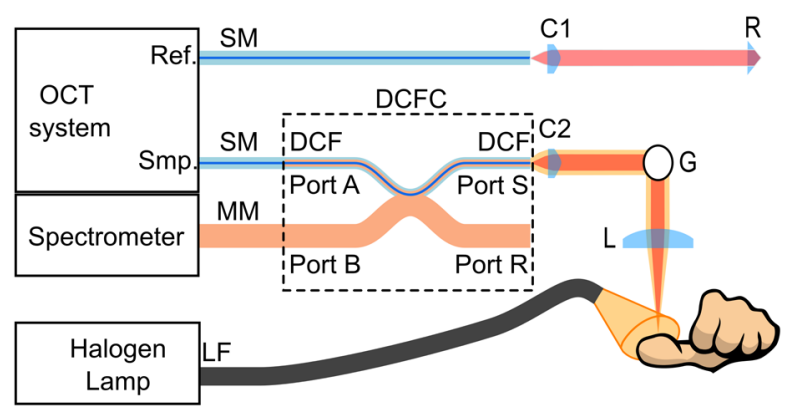

Fig. 1 Optical setup: Ref, reference arm port; Smp, sample arm port; SM, single-mode fiber; MM, multimode fiber; DCF, double-clad fiber; DCFC, double-clad fiber coupler; $\mathrm{C} 1$ and $\mathrm{C} 2$, collimation lenses; $\mathrm{G}$, dual-axis galvanometers; L, focalization lens; R, retroreflector prism; LF, light guide fiber. was used. The interference from the two arms is detected by a dual-balanced detector. The commercial OCT engine performs acquisition, processing, and display.

HSI acquisition is performed using an external white light source (MKII fiber optic light, Nikon Inc., New York). The broad illuminated area is sampled by imaging the face of the DCF (port S) onto the sample using two galvanometer-mounted mirrors. Light reflected from each point is collected primarily by the inner-cladding of the DCF due to the high area ratio between the inner-cladding and the core. Light from the inner-cladding is rerouted to an MM fiber by the DCFC and sent to a spectrometer (Maya2000, Ocean Optics, California). This spectrometer performs continuous 2064-point spectral measurements at a rate of $125 \mathrm{~Hz}$. Despite the spectrometer's bandwidth spanning from 200 to $1100 \mathrm{~nm}$, the useful bandwidth of the system spans from 450 to $800 \mathrm{~nm}$ due to the system's spectral response and the lamp's spectrum.

In the current implementation, HSI and OCT acquisitions are performed sequentially and the sample is immobilized to reduce potential motion artifacts throughout the acquisitions. Both OCT and HSI use the same scanning mechanism and share a field of view of $\sim 6 \times 6 \mathrm{~mm}^{2}$. Both the OCT source and the external halogen lamp are active during the acquisition to assess possible cross-talk between modalities. The built-in software of the commercial system is used to acquire the OCT data. HSI acquisition is realized with custom software (LabVIEW, National Instruments, Texas). The OCT volumes are $512 \times 512 \times$ 512 pixels and the acquisition is performed in less than $20 \mathrm{~s}$. The hyperspectral images are $75 \times 75$ pixels in size over the same field of view and were acquired in $45 \mathrm{~s}$. Additionally, 10 adjacent spectral pixels are binned together to increase signal-to-noise ratio and decrease the total size of the HSI data set, resulting in a spectral resolution of $4.3 \mathrm{~nm}$. The number of pixels acquired in HSI was limited to $75 \times 75$ to reduce acquisition time and potential motion artifacts.

\subsection{Image Processing}

The acquired reflectance spectra were corrected for background noise and for the spectral response of the system, using Eq. (1)

$S(\lambda)=\frac{I_{s}(\lambda)-I_{b}(\lambda)}{I_{r}(\lambda)-I_{b}(\lambda)}$,

where $S(\lambda), I_{s}(\lambda), I_{b}(\lambda)$, and $I_{r}(\lambda)$ are the corrected reflectance, the sample reflected intensity, the recorded intensity when no sample is placed in front of the objective, and the reflected intensity of a sample with a flat spectral response (Spectralon, Labsphere, New Hampshire). From the reflectance spectrum, it is possible to simulate the reflected spectral power distribution for any illumination condition and determine the color the sampled region would appear to the human eye. This conversion was achieved by transforming each HSI spectrum into standard Red Green Blue (sRGB) color coordinates ${ }^{34}$ using standard CIE1931 (CIE: International Commission on Illumination) spectrum-to-color coordinate transformation.

Beyond the simple appearance of the sample under a certain illumination, the HSI data can be used to perform molecular analysis of the sample. Dawson et al. ${ }^{35}$ showed, using a simplified skin model, that the logarithm of the inverse reflectance (LIR) is roughly proportional to the sum of the absorbance of the skin constituents [see Eq. (2)]. The same idea was used to 
highlight molecular constituents in the sample by producing images at wavelengths corresponding to absorption peaks of the target molecule.

$$
\operatorname{LIR}(\lambda)=-\log _{10}[S(\lambda)]
$$

\subsection{Combined Optical Coherence Tomography and Hyperspectral Imaging Volumetric Data}

The OCT and hyperspectral datasets were combined by overlaying the spectral information in sRGB format onto the surface of OCT slices. To obtain the surface topography from the OCT data, we implemented a custom surface detection algorithm based on the intensity and the first-order derivative of the signal as well as the relative position of the surface pixels to their closest neighbors. Surface detection was performed on the average of three adjacent B-scans further processed using a median filter in the axial direction. For each A-line, the OCT signal above the surface was set to zero. Processing all A-lines in this manner resulted in a topographic map of the sample.

As the HSI and OCT acquisitions shared the same scanning mechanism and field of view and the sample was tightly immobilized throughout the sequential acquisition, the output of both methods were coregistered. After resizing the RGB image produced from HSI data using bilinear interpolation, each individual color pixel was assigned to the corresponding A-line, then 7 pixels just above the surface axial position were colored using the assigned value from the RGB image. The number of pixels overlaid over the surface was chosen to improve visibility while minimizing the loss of topographic information. All algorithmic steps were performed using MATLAB ${ }^{\circledR}$.

\section{Results}

\subsection{System Performance Analysis}

In OCT, the numerical aperture of the beam in the sample space is $\sim 0.04$. This numerical aperture results in a theoretical lateral resolution of $13 \mu \mathrm{m}$ (defined as the $10 \%$ to $90 \%$ distance of the edge response for a Gaussian point spread function). The theoretical axial resolution of the OCT system remains unchanged with respect to the commercial system with a theoretical value of $12 \mu \mathrm{m}$ in air. Using the $10 \%$ to $90 \%$ distance of an edge response, the measured experimental lateral resolution in OCT is $14.5 \mu \mathrm{m}$. The experimental axial resolution in OCT is $17.4 \mu \mathrm{m}$, measured as the full width at half maximum of an A-line for a perfect reflector. The slight loss in axial resolution could be explained by a small dispersion mismatch between the sample and reference arm induced by the addition of the DCFC.

Assuming a uniform illumination distribution on a flat sample, the theoretical lateral resolution in HSI is given by the diameter of the image of the DCF inner-cladding on the sample and is $\sim 330 \mu \mathrm{m}$. The measured experimental lateral resolution for HSI is $345 \mu \mathrm{m}$. This resolution was measured using the $10 \%$ to $90 \%$ distance of an edge response on a sharp interface of a hyperspectral image.

\subsection{Combined Optical Coherence Tomography and Spectral Imaging}

A healing epithelial wound on the hand was imaged to evaluate the performance of the system. The subject rested his hand on a microscope slide to help reduce movement artifacts throughout both OCT and HSI acquisitions. The reference arm delay was adjusted to position the objective focal plane just passed the zero-delay plane of OCT. The microscope slide was then placed at the focus by positioning the hand surface a few hundred microns below the zero-delay plane. The two white-light illumination fibers were placed at an angle on each side of the sample to avoid the collection of specular reflection and provide a uniform illumination over the imaged area. A 3-D acquisition was then performed using the OCT engine, rapidly followed by HSI acquisition. OCT and HSI acquisitions could not currently be realized simultaneously due to differences in integration times associated with each modality, a challenge that would be lifted with custom-built systems.

Figure 2(a) shows an image of the healing wound taken with a CCD camera under ambient light conditions. Figure 2(b) displays a typical B-scan of the hand acquired with OCT. A maximum intensity projection was realized to produce en-face

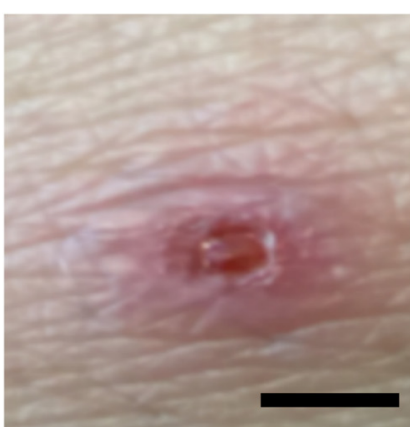

(a)

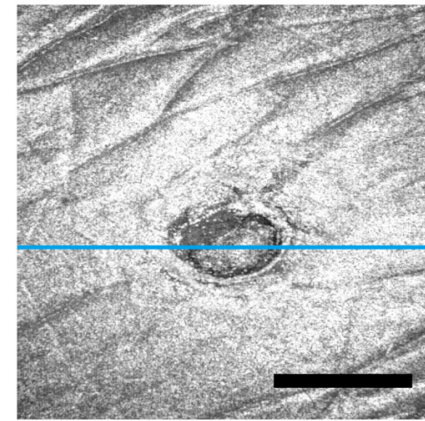

(c)

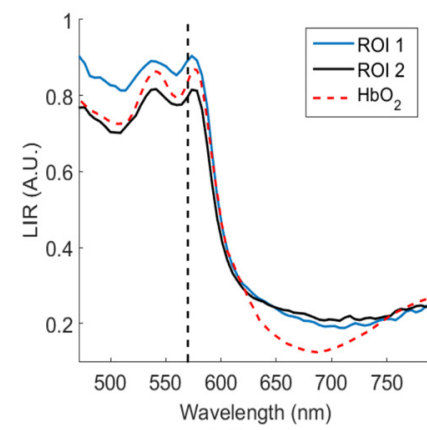

(e)

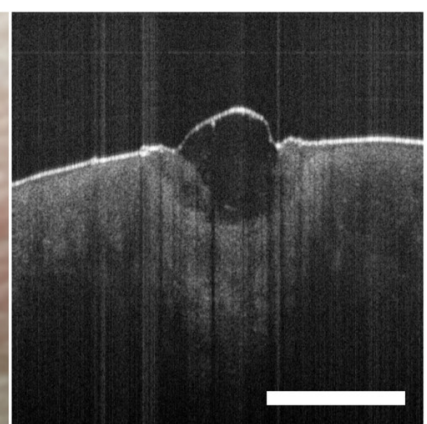

(b)

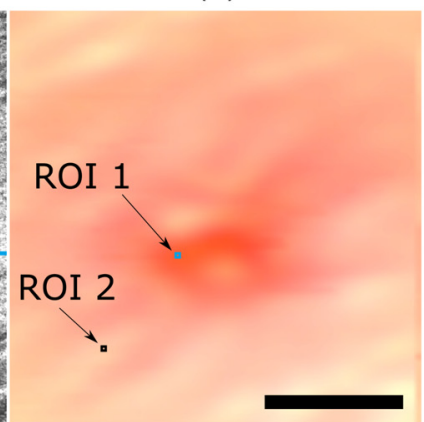

(d)

Fig. 2 OCT and HSI images from a healing epithelial wound: (a) ambient CCD image, (b) typical OCT B-scan, (c) en-face OCT reconstruction, (d) en-face RGB image reconstructed from HSI data, (e) LIR representation as a function of wavelength for two ROls identified in (d), (f) en-face LIR image at $575 \mathrm{~nm}$, identified as the dashed line in (e). Scale bars are $2 \mathrm{~mm}$. (b) Corresponds to the section-plane represented by the blue line in (c). 


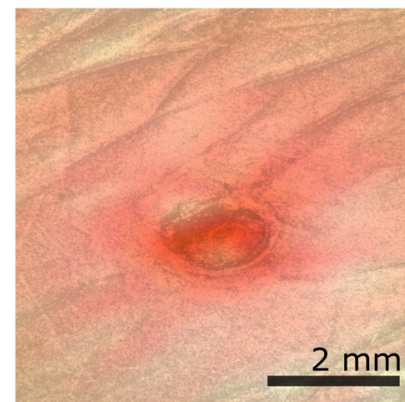

(a)

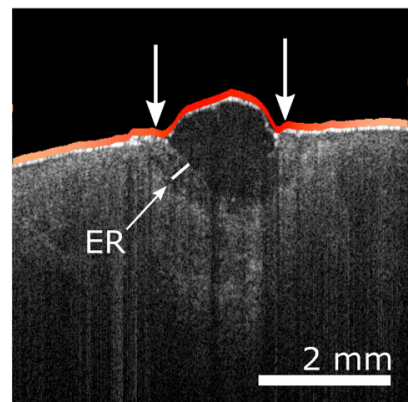

(b)

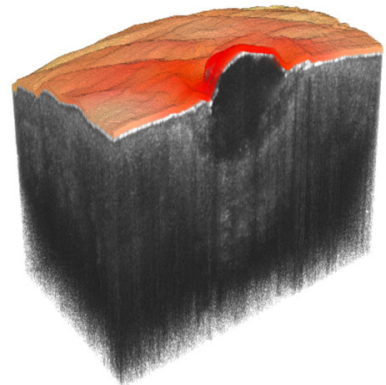

(c)

Fig. 3 Combining OCT and HSI data: (a) OCT en-face projection overlaid with RGB image, (b) OCT Bscan with $\mathrm{HSI}$ extracted true-color overlay. An epithelial region is forming in the wounded area (ER); (c) dual-modality 3-D rendering of the hand.

images from the OCT C-scan [Fig. 2(c)]. En-face true-color reconstruction was extracted from the HSI data [Fig. 2(d)]. Figure 2(e) plots the LIR representation as a function of wavelength in two regions of interest (ROIs) of the HSI image [shown in Fig. 2(d)] and a typical absorption spectrum of oxygen-saturated hemoglobin. ${ }^{36}$ Figure 2(e) shows that LIR representation is an adequate approximation to recover the absorption spectrum of the imaged tissue, which is, in this case, mostly composed of hemoglobin. From the absorption spectrum, one could retrieve the relative proportion of the main tissue constituents using spectral unmixing. ${ }^{37}$ Using the LIR representation, it is possible to detect specific absorption peaks in the sample and assess the presence of a specific absorber. For example, Fig. 2(f) shows the LIR image obtained at $575 \mathrm{~nm}$ [vertical line in Fig. 2(e)] which corresponds to an absorption peak in the hemoglobin absorption spectrum and gives insight on the distribution of hemoglobin in the sample. However, one should be careful interpreting Fig. 2(f) as it does not decouple the effect of the sample's topology on the absorption spectrum and some of the small features observed in Fig. 2(f) could be caused by shadows induced by the irregular surface of the sample.

Figure 3 shows the combination of OCT and HSI. Figure 3(a) was obtained by overlaying the RGB image [Fig. 2(d)] and the OCT en-face projection [Fig. 2(c)] and adjusting the transparency of both layers. A 7-pixel-wide region just above the surface of an OCT B-scan was colored using the true-color information obtained with HSI [Fig. 3(b)]. The same overlay can be applied in 3-D, as shown in Fig. 3(c).

\section{Discussion}

OCT is a powerful tool to describe the 3-D structure of a sample with high resolution. In the case of the healing epithelial wound, OCT provides the surface sample topology and the quantitative dimensions of the lesion. The OCT cross-sections enable the detection of what appears to be an epithelial regrowth just underneath the wound [indicated by ER on Fig. 3(b)]. Except for the general position and dimension of the wound, this information is not present in the HSI image. However, HSI enables the detection of what might be inflammation in the area surrounding the wound that is otherwise invisible in OCT, as indicated by the arrows above the surface of the tissue in Fig. 3(b). In future work, HSI data could be correlated to the sample's absorption, fluorescence, and scattering properties, thus giving access to quantitative diagnostic information about a given tissue's pathology ${ }^{38-41}$ and enabling molecular imaging of biological absorbers of interest such as hemoglobin, melanin, or other contrast agents (exogenous or endogenous).

The acquisition of a complete set of volumetric data requires about $65 \mathrm{~s}$. In order to be used in a clinical trial, the system should be able to acquire both the HSI and OCT data under a few seconds. A first improvement toward this goal would be to acquire both modalities simultaneously. This should be easy to address, as this only a software issue: no image degradation was observed due to cross-talk between the OCT and HSI channels since the working spectral bandwidths of HSI and OCT are more than $400 \mathrm{~nm}$ apart. Furthermore, the current HSI acquisition speed was limited by the spectrometer refresh rate $(125 \mathrm{~Hz})$. Line scan cameras with line rates higher than $100 \mathrm{kHz}$ are commercially available (Piranha4, Teledyne DALSA, Ontario, Canada) and would allow synchronous HSI and OCT acquisitions. Because computational complexity of HSI is small compared to the OCT signal processing, OCT images overlaid with HSI data could be displayed in real-time, and provide clinicians with valuable molecular information coregistered to the OCT structural data. Moreover, simultaneous acquisition would eliminate any potential motion-induced discrepancies between both modalities, making them naturally coregistered.

The imaging setup presented in this paper used an external light source. The proposed method could readily be applied in clinical endoscopic procedures that already employ white light to illuminate the sample. ${ }^{42-44}$ Alternatively, one could replace the external source with a white-light laser (e.g., a supercontinuum laser) injected in the DCF core for HSI illumination. This would ensure a localized illumination and would help avoid the creation of shadows from the endoscope or the sample. Light throughput would also be improved as the illumination would be restricted to the RIO. Furthermore, illumination through the DCF core would eliminate the need for the external light source and enable the fabrication of sub-1-mm-diameter endoscopes combining OCT and HSI, as proven by other multimodal endoscope based on DCF fibers. ${ }^{31,32}$

The experimental lateral resolution of HSI is $\sim 345 \mu \mathrm{m}$, which is quite coarse compared to the lateral resolution in OCT. The HSI lateral resolution could be improved by decreasing the size of the HSI collection spot (clad image on sample), but that would require increasing the OCT numerical aperture and consequently decreasing the OCT depth of field. Illuminating the sample using the fiber core, as proposed earlier, would reduce the size of the volume probed by the white-light, hence increasing the HSI resolution. In this case, the resolution would now be 
limited by size of the illumination spot on the sample while still enabling the collection of the scattered photons in the cladding.

\section{Conclusion}

In this work, we demonstrated the first combined OCT and HSI images collected via a single-optical fiber using a DCFC. The proposed method could be miniaturized into an endoscope with a diameter as small as $1 \mathrm{~mm} .{ }^{45}$ The clinical potential of the combination of OCT and HSI arises from both modalities bringing complementary views of the sample: the first one giving access to sample morphology and the second to sample molecular composition. Their combination in a clinical setting could improve current diagnostic practice. For example, the information about remodeling due to a pathology (observed in OCT) could be merged with the change in molecular composition (observed in HSI), in order to allow earlier detection or more accurate diagnostics. HSI could also be used to infer the oxy/deoxyhemoglobin ratio in combination with the ability of OCT to observe vessels and quantify blood flow ${ }^{26}$ for a complete characterization of tissue perfusion. While future work using an improved simultaneous and real-time system is needed to demonstrate the usefulness of the technique in a clinical context, the encouraging preliminary results presented in this paper illustrates the potential of the combination of OCT and HSI using a DCFC for biomedical applications.

\section{Acknowledgments}

This work was funded by the Canada Foundation for Innovation (CFI), the Natural Sciences and Engineering Research Council of Canada (NSERC), the Research Group in Biomedical Sciences and Technologies (GRSTB), and by the National Science Foundation Graduate Research Fellowship (NSFGRF). This work is an expanded and refined version of a proceeding published for a poster session at Photonics West $2016 .{ }^{46}$ Imaging of the finger of a healthy volunteer was approved by Ecole Polytechnique's Ethics Committee under project \#BIO-08/09-01.

\section{References}

1. P. Hrynchak and T. Simpson, "Optical coherence tomography: an introduction to the technique and its use," Optom. Vis. Sci. 77, 347-356 (2000).

2. E. Regar et al., "Optical coherence tomography," Cardiovasc. Radiat. Med. 4, 198-204 (2003).

3. J. A. Burns, "Optical coherence tomography: imaging the larynx," Curr. Opin. Otolaryngol. Head Neck Surg. 20, 477-481 (2012).

4. M. J. Gora et al., "Tethered capsule endomicroscopy enables less invasive imaging of gastrointestinal tract microstructure," Nat. Med. 19, 238-240 (2013).

5. T.-H. Tsai, J. Fujimoto, and H. Mashimo, "Endoscopic optical coherence tomography for clinical gastroenterology," Diagnostics 4, 57-93 (2014).

6. H. Nakagawa et al., "Validation of swept source optical coherence tomography (SS-OCT) for the diagnosis of smooth surface caries in vitro," J. Dent. 41, 80-89 (2013).

7. W. Wieser et al., "High definition live 3D-OCT in vivo: design and evaluation of a 4D OCT engine with $1 \mathrm{GVoxel} / \mathrm{s}$," Biomed. Opt. Express 5, 2963 (2014).

8. G. Lu and B. Fei, "Medical hyperspectral imaging: a review," J. Biomed. Opt. 19, 010901 (2014).

9. M. E. Martin et al., "Development of an advanced hyperspectral imaging (HSI) system with applications for cancer detection," Ann. Biomed. Eng. 34, 1061-1068 (2006).

10. H. Akbari et al., "Hyperspectral imaging and quantitative analysis for prostate cancer detection," J. Biomed. Opt. 17, 076005 (2012).

11. S. Kiyotoki et al., "New method for detection of gastric cancer by hyperspectral imaging: a pilot study," J. Biomed. Opt. 18, 026010 (2013).
12. D. Yudovsky, A. Nouvong, and L. Pilon, "Hyperspectral imaging in diabetic foot wound care," J. Diabetes Sci. Technol. 4, 1099-1113 (2010).

13. H. Akbari et al., "Detection and analysis of the intestinal ischemia using visible and invisible hyperspectral imaging," IEEE Trans. Biomed. Eng. 57, 2011-2017 (2010).

14. M. S. Holzer et al., "Assessment of renal oxygenation during partial nephrectomy using hyperspectral imaging," J. Urol. 186, 400-404 (2011).

15. W. R. Johnson et al., "Snapshot hyperspectral imaging in ophthalmology," J. Biomed. Opt. 12, 014036 (2007).

16. D. Cohen et al., "The use of spectral imaging for the diagnosis of retinal disease," in 1999 IEEE LEOS Annual Meeting Conf. Proc. (LEOS 1999). 12th Annual Meeting. IEEE Lasers and Electro-Optics Society 1999 Annual Meeting (Cat. No. 99CH37009), IEEE, Vol. 1, pp. 220221 (1999).

17. J. Schweizer et al., "Hyperspectral imaging — a new modality for eye diagnostics," Biomed. Eng. 57, 293-296 (2012).

18. S. T. Monteiro et al., "Towards applying hyperspectral imagery as an intraoperative visual aid tool," in Proc. 4th IASTED Int. Conf. on Visualization, Imaging and Image Processing (VIIP), Marbella, Spain, pp. 483-488 (2004).

19. S. V. Panasyuk et al., "Medical hyperspectral imaging to facilitate residual tumor identification during surgery," Cancer Biol. Ther. 6, 439-446 (2007).

20. K. J. Zuzak et al., "Active DLP hyperspectral illumination: a noninvasive, in vivo, system characterization visualizing tissue oxygenation at near video rates," Anal. Chem. 83, 7424-7430 (2011).

21. U. Morgner et al., "Spectroscopic optical coherence tomography," Opt. Lett. 25, 111 (2000).

22. C. P. Fleming et al., "Depth resolved detection of lipid using spectroscopic optical coherence tomography," Biomed. Opt. Express 4, 1269 (2013).

23. F. E. Robles et al., "Molecular imaging true-colour spectroscopic optical coherence tomography," Nat. Photonics 5, 744-747 (2011).

24. D. J. Faber et al., "Light absorption of hemoglobin assessed by spectroscopic optical coherence tomography," Opt. Lett. 28, 1436-1438 (2003).

25. N. Bosschaart et al., "Quantitative comparison of analysis methods for spectroscopic optical coherence tomography," Biomed. Opt. Express 4, 2570 (2013).

26. M. C. Skala et al., "Combined hyperspectral and spectral domain optical coherence tomography microscope for noninvasive hemodynamic imaging," Opt. Lett. 34, 289-291 (2009).

27. P. Zhang et al., "In vivo wide-field multispectral scanning laser ophthalmoscopy-optical coherence tomography mouse retinal imager: longitudinal imaging of ganglion cells, microglia, and Müller glia, and mapping of the mouse retinal and choroidal vasculature," J. Biomed. Opt. 20, 126005 (2015).

28. S. Lemire-Renaud et al., "Double-clad fiber coupler for endoscopy," Opt. Express 18, 9755 (2010).

29. W.-J. Madore et al., "Asymmetric double-clad fiber couplers for endoscopy," Opt. Lett. 38, 4514 (2013).

30. E. De Montigny et al., "Double-clad fiber coupler for partially coherent detection," Opt. Express 23, 9040-9051 (2015).

31. D. Lorenser et al., "Dual-modality needle probe for combined fluorescence imaging and three-dimensional optical coherence tomography," Opt. Lett. 38, 266 (2013).

32. L. Scolaro et al., "Molecular imaging needles: dual-modality optical coherence tomography and fluorescence imaging of labeled antibodies deep in tissue," Biomed. Opt. Express 6, 1767-1781 (2015).

33. K. Beaudette et al., "Laser tissue coagulation and concurrent optical coherence tomography through a double-clad fiber coupler," Biomed. Opt. Express 6, 1293-1303 (2015).

34. R. W. G. Hunt, W. G. Robert, and M. Pointer, Measuring Colour, Wiley, Hoboken, New Jersey (2011).

35. J. B. Dawson et al., "A theoretical and experimental study of light absorption and scattering by in vivo skin," Phys. Med. Biol. 25, 695-709 (1980).

36. S. Prahl, "Optical absorption of hemoglobin," 1999, Oregon Medical Laser Center, http://omlc.org/spectra/hemoglobin/ (17 May 2016).

37. N. Keshava and J. F. Mustard, "Spectral unmixing," IEEE Signal Process. Mag. 19, 44-57 (2002). 
38. C. Balas, C. Pappas, and G. Epitropou, Handbook of Biomedical Optics, pp. 131-164, CRC Press, Boca Raton, Florida (2011).

39. V. Tuchin, Tissue Optics, SPIE, Bellingham, Washington (2007).

40. D. G. Ferris et al., "Multimodal hyperspectral imaging for the noninvasive diagnosis of cervical neoplasia," J. Low. Genit. Tract Dis. 5, 65-72 (2001).

41. M. C. Pierce et al., "Accuracy of in vivo multimodal optical imaging for detection of oral neoplasia," Cancer Prev. Res. 5, 801-809 (2012).

42. M. Tsuboi et al., "Optical coherence tomography in the diagnosis of bronchial lesions," Lung Cancer 49, 387-394 (2005).

43. Y. Fradet et al., "A comparison of hexaminolevulinate fluorescence cystoscopy and white light cystoscopy for the detection of carcinoma in situ in patients with bladder cancer: a phase III, multicenter study," J. Urol. 178, 68-73 (2007).
44. G. Tao et al., "Enhanced magnifying endoscopy for differential diagnosis of superficial gastric lesions identified with white-light endoscopy," Gastric Cancer 17, 122-129 (2014).

45. K. L. Lurie et al., "Rapid scanning catheterscope for expanded forwardview volumetric imaging with optical coherence tomography," Opt. Lett. 40, 3165 (2015).

46. G.-L. Robin et al., "Combined optical coherence tomography and hyper-spectral imaging using a double clad fiber coupler," Proc. SPIE 9701, 97010X (2016).

Biographies for the authors are not available. 